\title{
C-Myc suppresses miR-451 1 YWTAZ/AKT axis via recruiting HDAC3 in acute myeloid leukemia
}

\author{
Rui Su${ }^{1}$, Jia-Nan Gong ${ }^{1}$, Ming-Tai Chen ${ }^{1}$, Li Song ${ }^{1}$, Chao Shen ${ }^{1}$, Xin-Hua Zhang ${ }^{2}$, \\ Xiao-Lin Yin ${ }^{2}$, Hong-Mei Ning ${ }^{3}$, Bing Liu ${ }^{4}$, Fang Wang ${ }^{1}$, Yan-Ni Ma ${ }^{1}$, Hua-Lu Zhao ${ }^{1}$, \\ Jia Yu ${ }^{1}$, Jun-Wu Zhang ${ }^{1}$
${ }^{1}$ The State Key Laboratory of Medical Molecular Biology, Department of Biochemistry and Molecular Biology, Institute of Basic Medical Sciences, Chinese Academy of Medical Sciences and Peking Union Medical College, Beijing, China
${ }^{2}$ Department of Hematology, The 303 Hospital, Nanning, Guangxi, China
${ }^{3}$ Department of Hematopoietic Stem Cell Transplantation, Affiliated Hospital to Academy of Military Medical Sciences, The 307 Hospital, Beijing, China
${ }^{4}$ State Key Laboratory of Proteomics, Translational Medicine Center of Stem Cells, 307-Ivy Translational Medicine Center, Laboratory of Oncology, Affiliated Hospital of Academy of Military Medical Sciences, Beijing, China

Correspondence to: Jun-Wu Zhang, email: junwu_zhang@pumc.edu.cn

Keywords: acute myeloid leukemia, microRNA-451, c-Myc, HDAC3, YWHAZ

Received: February 20, 2016

Accepted: September 20, 2016

Published: October 15, 2016

\section{ABSTRACT}

\begin{abstract}
Aberrant activation of c-Myc plays an important oncogenic role via regulating a series of coding and non-coding genes in acute myeloid leukemia (AML). Histone deacetylases (HDACs) can remove acetyl group from histone and regulate gene expression via changing chromatin structure. Here, we found miR-451 is abnormally down-regulated in AML patient samples; c-Myc recruits HDAC3 to form a transcriptional suppressor complex, co-localizes on the miR-451 promoter, epigenetically inhibits its transcription and finally induces its downregulation in AML. Furthermore, our in vitro and in vivo results suggest that $\mathbf{m i R}-\mathbf{4 5 1}$ functions as a tumor suppressor via promoting apoptosis and suppressing malignant cell proliferation. The mechanistic study demonstrated that miR-451 directly targets $Y W H A Z$ mRNA and suppresses YWHAZ/AKT signaling in AML. Knockdown of $c-M y c$ results in restoration of miR451 and inhibition of YWHAZ/AKT signaling. In AML patients, low level of miR-451 is negatively correlated with high levels of c-Myc and YWHAZ, while c-Myc level is positively related to YWHAZ expression. These results suggested that $\mathrm{C}-\mathrm{Myc}-1 \mathrm{miR}-$ $451 \dashv$ YWHAZ/AKT cascade might play a crucial role during leukemogenesis, and reintroduction of miR-451 could be as a potential strategy for AML therapy.
\end{abstract}

\section{INTRODUCTION}

Acute myeloid leukemia (AML) is a heterogenous disorder of aggressive hematopoietic disease characterized by malignant proliferation of clonal neoplastic cells and differentiation arrest of myeloid blasts [1-3]. It is widely reported that Notch signaling [4], PI3K/AKT pathway [5], JUN and c-Myc are indispensable and critical for leukemiagenesis [6-9]. AML-associated fusion proteins, such as AML-ETO and PML/RARA, could induce expression of $c-M y c$ [10]. Hyperactivation of $c-M y c$ is one of the most frequent events associated with AML and c-Myc is essential for cell growth, hepatopoiesis differentiation and leukemogenesis [11]. Histone deacetylases (HDACs) are involved in remodeling nucleosomes and chromatin via removing acetyl group from histone and function as critical transcriptional co-repressors in epigenetic regulation of gene expression [12, 13]. Interestingly, HDAC families are abnormally up-regulated in leukemia [14] and HDAC inhibitors have been used to treat malignant leukemia in clinical studies [15-17]. However, the synergetic regulation of c-Myc and HDAC is not clearly studied in AML.

MicroRNAs (miRNAs), a class of small noncoding RNA, are emerging as important posttranscriptional players during normal hematopoiesis and deregulation of specific miRNAs are associated with initiation, progression, diagnosis as well as prognosis of leukemia [18-20]. As hematopoietic differentiation, miR-451 is required for erythroid homeostasis and plays a crucial role in promoting erythroblast maturation [21, 22]. MiR-451 
also functions as a tumor suppressor via targeting $c-M y c$ mRNA in lung adenocarcinoma [23], IKK- $\beta$ mRNA in

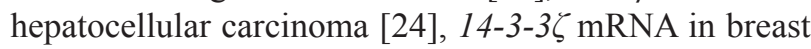
cancer [25]. However the expression level, the potential function of miR-451 and its correlation with c-Myc and HDAC family is not clear yet in AML.

Here, we found that hyperactivation of c-Myc could recruit HDAC3 to bind on the promoter region of $m i R-451$ and induce the deacethylation of histone, which finally results in deregulation of $m i R-451$ in AML. Moreover, in vitro and in vivo reintroduction of miR-451 could promote cell apoptosis and inhibit uncontrolled proliferation via directly targeting YWHAZ/ AKT signaling.

\section{RRSULTS}

\section{miR-451 is abnormally down-regulated in AML patients}

We analyzed relative expression of miR-451 in peripheral blood $(\mathrm{PB})$ mononuclear cells (MNCs) derived from 69 primarily diagnosed AML patients with different mutation and genomic translocation (Supplementary Table $\mathrm{S} 1$ ) and 80 healthy donors. The data indicated that miR451 is abnormally down-regulated in the AML patients (Figure 1A). The receiver-operating characteristic (ROC) curve result suggested that expression level of miR-451 could be as a marker with high sensitivity and specificity for AML diagnosis (Figure 1B). Similarly, as shown in Figure $1 \mathrm{C}$ and $1 \mathrm{E}$, the relative expression of miR-451 was also significantly suppressed in bone marrow (BM) MNCs and $\mathrm{BM} \mathrm{CD} 34^{+}$hematopoietic stem/progenitor cells (HSPCs) of AML patients. Moreover, the ROC curves indicated that miR-451 level could also be as important marker for AML diagnosis in BM MNC samples and CD34 ${ }^{+}$HSPCs samples (Figure 1D and 1F). We did not observe significant difference of miR-451 expression among the AML FAB subtypes or cytogenetically normal AML (CN-AML) vs cytogenetically abnormal AML (CAAML) (Supplementary Figure S1A-S1D). These results suggest miR-451 might function as a tumor suppressor in AML development.

\section{c-Myc directly suppresses miR-451 expression by recruiting HDAC3 in AML}

To understand how miR-451 is downregulated in AML patients, we used bioinformatics to analyze $2500 \mathrm{bp}$ upstream of $m i R-451$ to see whether it contains potential binding sites for transcriptional factors that are involved in leukemogenesis. Interestingly, we identified multiple nonconsensus E box elements, which may be involved in potential binding sites of some transcriptional factors including $\mathrm{c}-\mathrm{Myc}$, on the $m i R-451$ promoter region. Our chromatin Immunoprecipitation (ChIP)-PCR showed that
c-Myc could bind to one of these potential binding sites, which is located at -158 bp upstream of miR-451 (Figure $2 \mathrm{~A}$ ), in both NB4 and HL-60 AML cells (Figure 2B and $2 \mathrm{C})$. To evaluate the effect of c-Myc activity on miR-451 expression, we constructed miR-451 promoter-reporter vectors containing wild-type or deleted c-Myc binding site for dual-luciferase reporter assay. The data indicated that forced expression of $c-M y c$ could significantly inhibit the transcriptional activity of $m i R-451$ promoter, while deletion of the c-Myc binding site abolished this suppression in HEK-293T cells (Figure 2D). Moreover, knockdown of $c-M y c$ in both NB4 and HL-60 cell lines by siRNAs transfection could promoted expression of miR-451 (Figure 2E). Our results suggested that c-Myc directly binds on the $m i R-451$ promoter and suppresses its expression, which may be one of the most important reasons which result in its deregulation in AML.

As reported, c-Myc could recruit HDAC1 or HDAC3 to suppress expression of specific genes, such as HPP1 [26], miR-29 [27], miR-15/16-1 [28], which leads us to investigate whether the recruitment of HDAC1/3 is involved in the transcriptional repression of $m i R-451$ by c-Myc. To address this hypothesis, we firstly examined effects of deacetylase inhibitor Trichostatin A (TSA) on miR-451 expression. As shown in Figure 2F, TSA caused a dose-dependent increase of miR-451 expression in NB4 and HL-60 AML cells, which suggested the role of HDACs in miR-451 expression. Our Co-immunoprecipitation (Co-IP) results indicated that HDAC3, but not HDAC1, physiologically interacted with c-Myc in the AML cells (Figure 2G). Importantly, ChIP-PCR showed that HDAC3 could co-localize with c-Myc to the same region on the miR-451 promoter (Figure $2 \mathrm{H}$ and 2I). And knockdown of $H D A C 3$ by siRNAs could increase miR-451 expression in NB4 and HL-60 AML cells (Figure 2J).

Taken together, our mechanistic data suggest that c-Myc recruits HDAC3 to co-localize on the $m i R-451$ promoter region, and repress its expression in AML.

\section{Forced expression of miR-451 decreases cell proliferation and increases cell apoptosis in AML}

The above results show that c-Myc negatively regulates $m i R-451$ via recruiting HDAC3 on the promoter region of $m i R-451$ and results in its deregulation in AML. We then focused on the function of miR-451 in AML. To this aim, we first transfected miR-451 mimics into HL-60 and NB4 cells and detected its effect on cell proliferation and apoptosis. The over-presence of miR-451 in the HL60 cells transfected with miR-451 mimics was confirmed (Figure 3A) by qPCR. Enforced expression of miR-451 significantly suppressed cell proliferation (Figure 3B) and induced cell apoptosis (Figure 3C) in the HL-60 cells. Similarly, forced expression of miR-451 (Figure $3 \mathrm{D})$ also restrained cell growth (Figure $3 \mathrm{E}$ ) and promoted cell apoptosis (Figure 3F) in the NB4 cells. While forced 
expression of miR-451 has little effects on cell cycle in AML cells (data not shown).

\section{Reintroduction of miR-451 significantly inhibits engraftment of leukemic cells and accelerates cell apoptosis in vivo}

To further understand the anti-leukemia function of miR-451 and examine the potential possibility that reintroduction of miR-451 for AML treatment, we constructed the AML murine model via injection NB4 cells into NOD/SCID mice for in vivo study. As compared to the control group injected with Lenti-GFP, reintroduction of miR-451 mediated by Lenti-miR-451 injection significantly inhibited splenomegaly induced by leukemia in AML NOD/SCID mice (Figure 4A, left). The infection efficiency and enforced expression of miR-451 were confirmed in BM and spleen samples (Figure 4A, middle and right panel; Supplementary Figure S2A-S2C). Hematoxylin and eosin (H \& E) staining showed that neoplastic infiltration was suppressed by Lenti-miR-451 in spleen (Figure 4B). More importantly, flow cytometry results strongly demonstrated that reintroduction of miR451 dramatically inhibited engraftment of leukemic cells into BM (Figure 4C; Figure 4F, left panel) and spleens (Figure 4C; Figure 4G, left panel). Forced expression of miR-451 also induced apoptosis both in BM (Figures 4D; Figure 4F, middle and right panels) and spleens (Figures 4E; Figure 4G, middle and right panels).

Totally, our in vivo data also suggest miR-451 functions as a tumor suppressor via regulating cell proliferation and apoptosis, and reintroduction of miR-451 could partially release leukemic symptoms in AML.

\section{miR-451 directly suppresses YWHAZ-AKT signaling in AML cells}

To determine the mechanism by which miR-451 regulates cell apoptosis and proliferation in AML cells, we tried to identify the potential targets of miR-451 via several
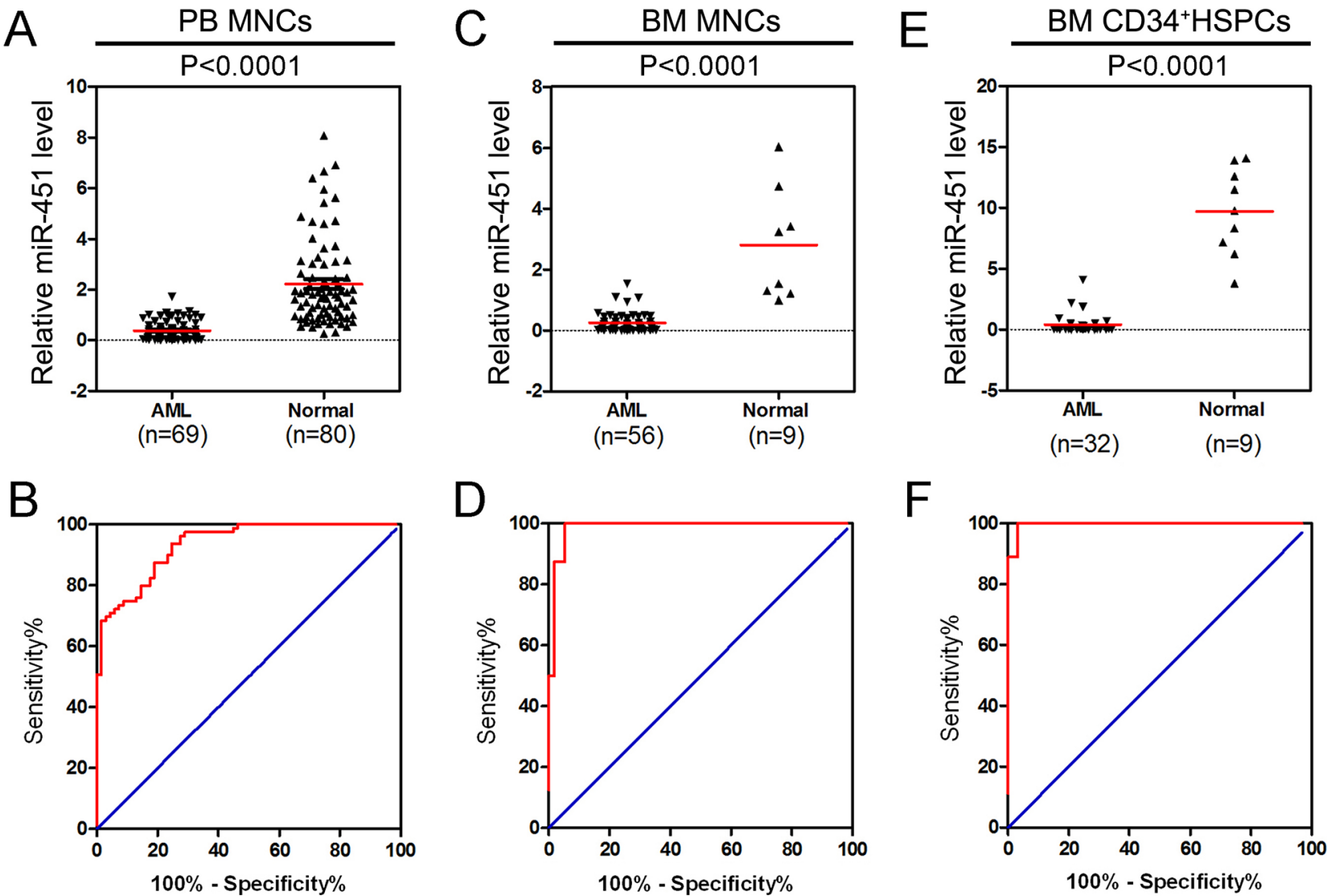

Figure 1: miR-451 is abnormally down-regulated in AML patients. A. The expression of miR-451 was determined by stem-loop real-time-PCR in PB MNCs derived from 69 AML patients and 80 healthy donors. U6 snRNA was used as the internal control and each real-time PCR assay was performed in triplicate. B. ROC curve analysis of miR-451 expression in the PB MNC samples. The area under ROC curve (AUC), sensitivity and specificity were $93.5 \%, 98.7 \%$ and $98.5 \%$, respectively. C. The relative level of miR-451 in BM MNCs from 56 AML patients and 9 healthy donors. D. ROC curve analysis of miR-451 expression in the BM MNC samples. The AUC, sensitivity and specificity were $98.7 \%, 87.5 \%$ and $98.2 \%$, respectively. E. The relative expression of miR-451 in BM CD $34^{+}$HSPCs from 38 AML patients and 9 normal controls. F. ROC curve analysis of miR-451 expression in CD34 ${ }^{+}$HSPCs. The AUC, sensitivity and specificity were $99.7 \%, 88.9 \%$ and $96.7 \%$, respectively. 
prediction programs and noticed that 3'UTR of YWHAZ mRNA contains the sequence motif that could match well with the "seed sequence" of $m i R-451$ (Figure 5A). The dual-luciferase reporter assay in HEK-293 cells indicated that forced expression of miR-451 significantly suppresses the activity of construct with wild type YWHAZ 3'UTR, but not mutant (Figure 5B). Additionally, miR-451 overexpression also inhibited $Y W H A Z$ expression both at RNA and protein levels (Figure 5C and 5D) in NB4 and HL-60 AML cells.

YWHAZ, also known as 14-3-3-zeta, is a member of 14-3-3 family and could mediate signal transduction by regulating phosphorylation of specific proteins [29]. Here, we found that enforced expression of miR-451
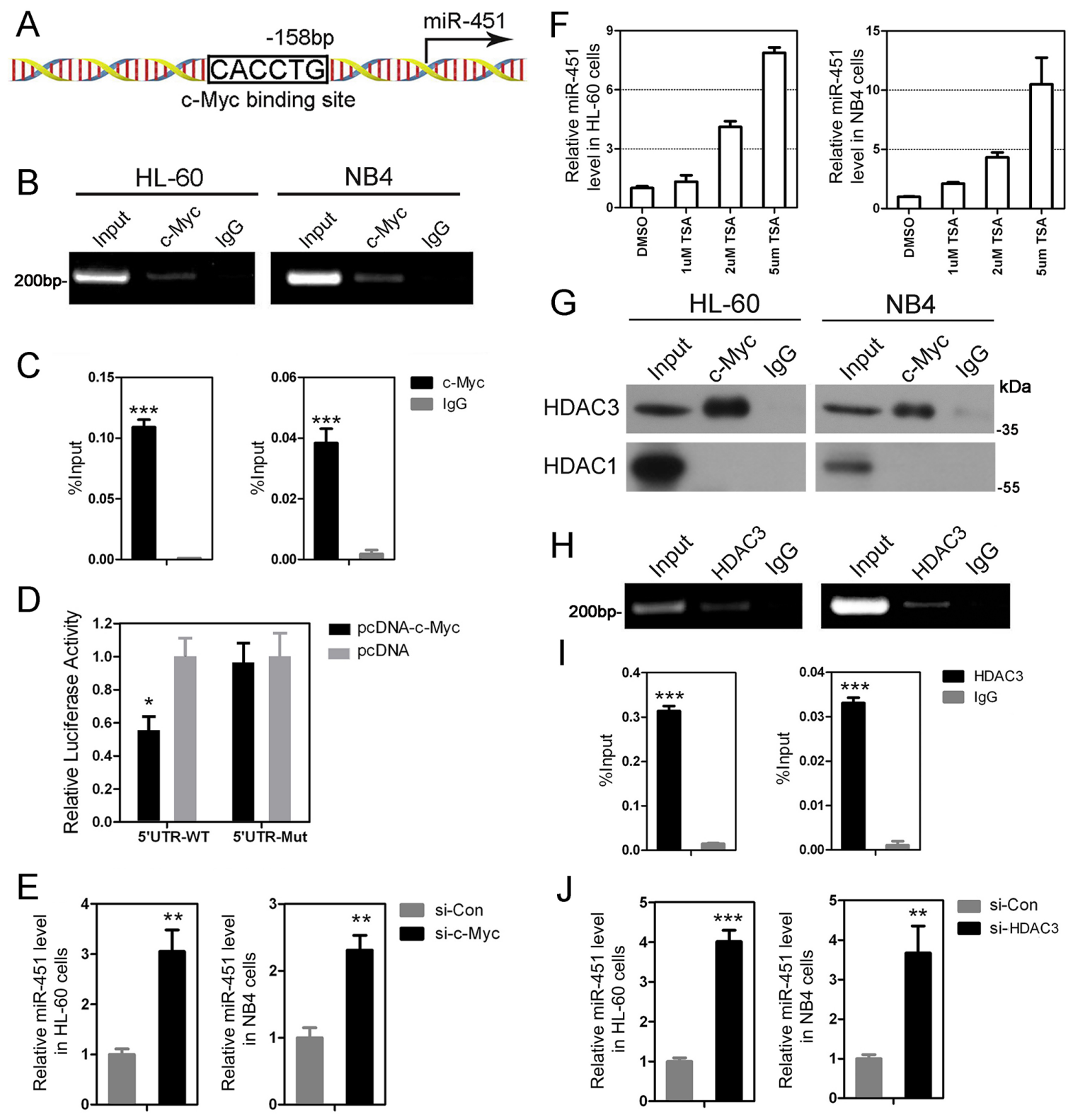

Figure 2: c-Myc suppresses expression of $\boldsymbol{m i R}-451$ via recruiting HDAC3. A. A sketch showing the c-Myc binding site at -158 bp upstream of the miR-451 promoter. The other potential binding sites, which are not been confirmed by ChIP-PCR, are not shown. B. The ChIP-PCR data indicate that c-Myc binds on the c-Myc binding site at -158 bp upstream of the miR-451 promoter in HL-60 and NB4 cells. C. Quantitative ChIP-PCR demonstrates a significant enrichment of c-Myc on the promoter region of $m i R-451$ in the AML cells. D. Enforced expression of c-Myc by pcDNA-c-Myc tensfection inhibits the activity of $m i R-451$ promoter. The dual-luciferase reporter assay was performed in triplicate in HEK-293 cells. Error bars represent SD. ${ }^{*} P<0.05$; Student's t-test. E. Knowdown of $c-M y c$ by si-c-Myc transfection increased miR-451 level in HL-60 and NB4 cells. ${ }^{* *} P<0.01$. F. TSA treatment significantly induces expression of miR-451 in the AML cells at a dose-dependent manner. G. The Co-IP assay indicates that c-Myc can interact with HDAC3, but not HDAC1, in H160 and NB4 cells. H. ChIP-PCR shows HDAC3 binds on the same location of $m i R-451$ promoter with c-Myc. I. Quantitative ChIP-PCR analysis demonstrated a significant enrichment of HDAC3 on the promoter region of $m i R-451$ in the AML cells. $* * * P<0.001$. J. Inhibition of HDAC3 by si-HDAC3 transfection dramatically accelerates expression of miR-451 in the AML cells. $* * P<0.01 ; * * * P<0.001$. 
significantly reduced phosphorylated AKT (p-AKT) level, but not total AKT, via targeting YWHAZ RNA in the AML cells (Figure 5D). Functionally, knockdown of $Y W H A Z$ increased cell apoptosis and suppressed cell proliferation (Figure 5E and 5F), suggesting that knockdown of $Y W H A Z$ could mimic the effects of miR-451 overexpression in the AML cells. Knockdown of YWHAZ reduced p-AKT level (Figure 5G, left panel), and knockdown of $c-M y c$ suppressed YWHAZ/AKT signaling via activating miR451 expression (Figure 5G, right panel). c-Myc inhibitor treatment also enhanced cell apoptosis in AML (Figure $5 \mathrm{H})$. Importantly, our rescue assay demonstrated that knockdown of $Y W H A Z$ could significantly reduce the high level of YWHAZ induced by anti-451 treatment (Figure

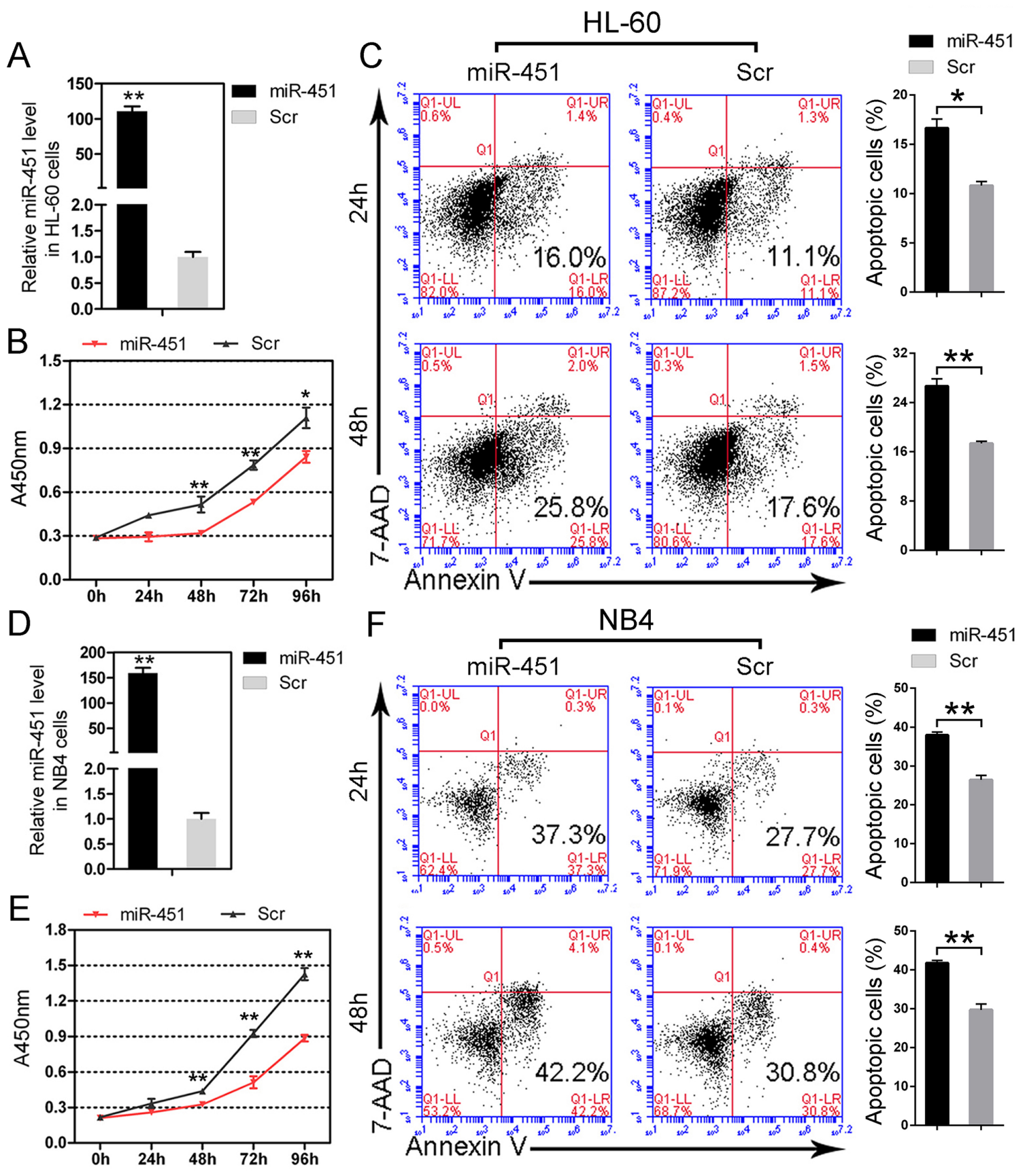

Figure 3: miR-451 restricts proliferation and induces apoptosis in AML cells. A. The over-presence of miR-451 was confirmed by qPCR in HL-60 cells transfected with miR-451 mimics. B. Enforced expression of miR-451 inhibits proliferation of HL-60 cells. C. Enforced expression of miR-451 accelerates serum deprivation-induced apoptosis in HL-60 cells. A representative experiment is presented on the left and a statistical analysis of three independent experiments on the right. Error bars represent SD. $* P<0.05 ; * * P<0.01$; Student's t-test. D. The over-presence of miR-451 was confirmed by qPCR in NB4 cells transfected with miR-451 mimics. E. Ectopic expression of miR-451 suppresses cell proliferation in NB4 cells. F. Overexpression of miR-451 promotes apoptosis in NB4 cells. A representative experiment is presented on the left and a statistical analysis of three independent experiments on the right. $* * P<0.01$. 
A
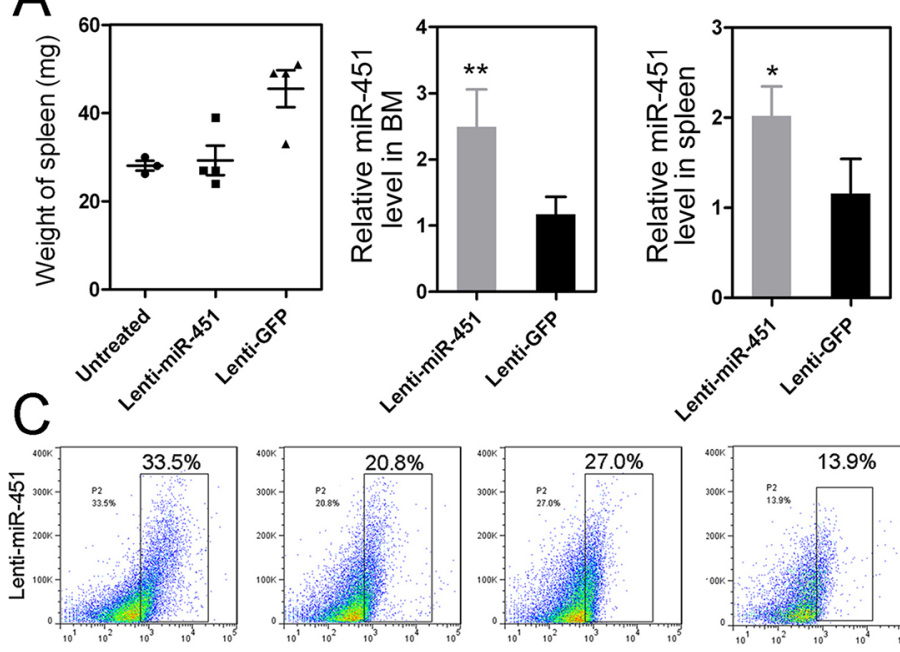

B
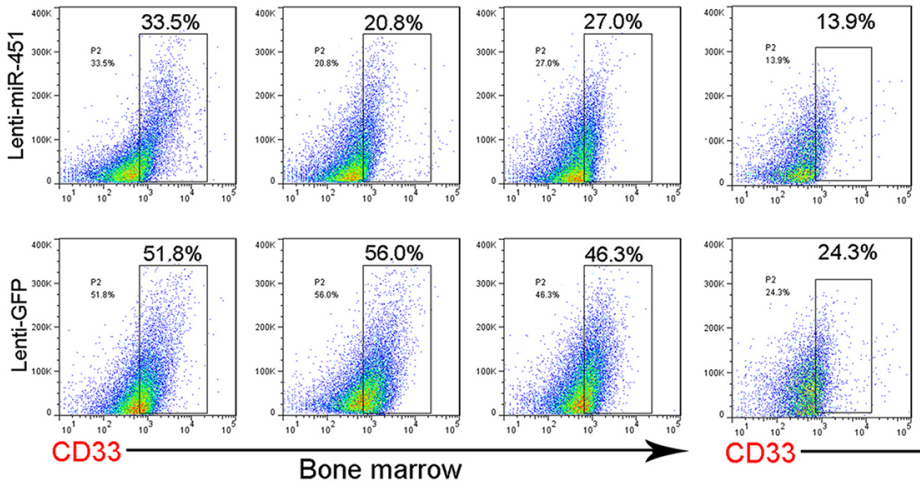

D

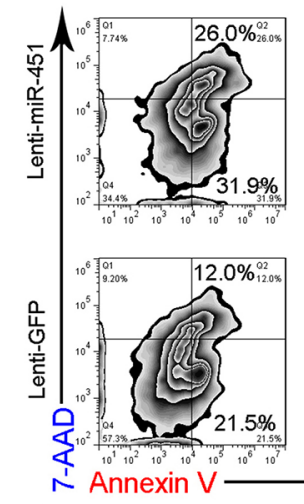

$\mathrm{F}$
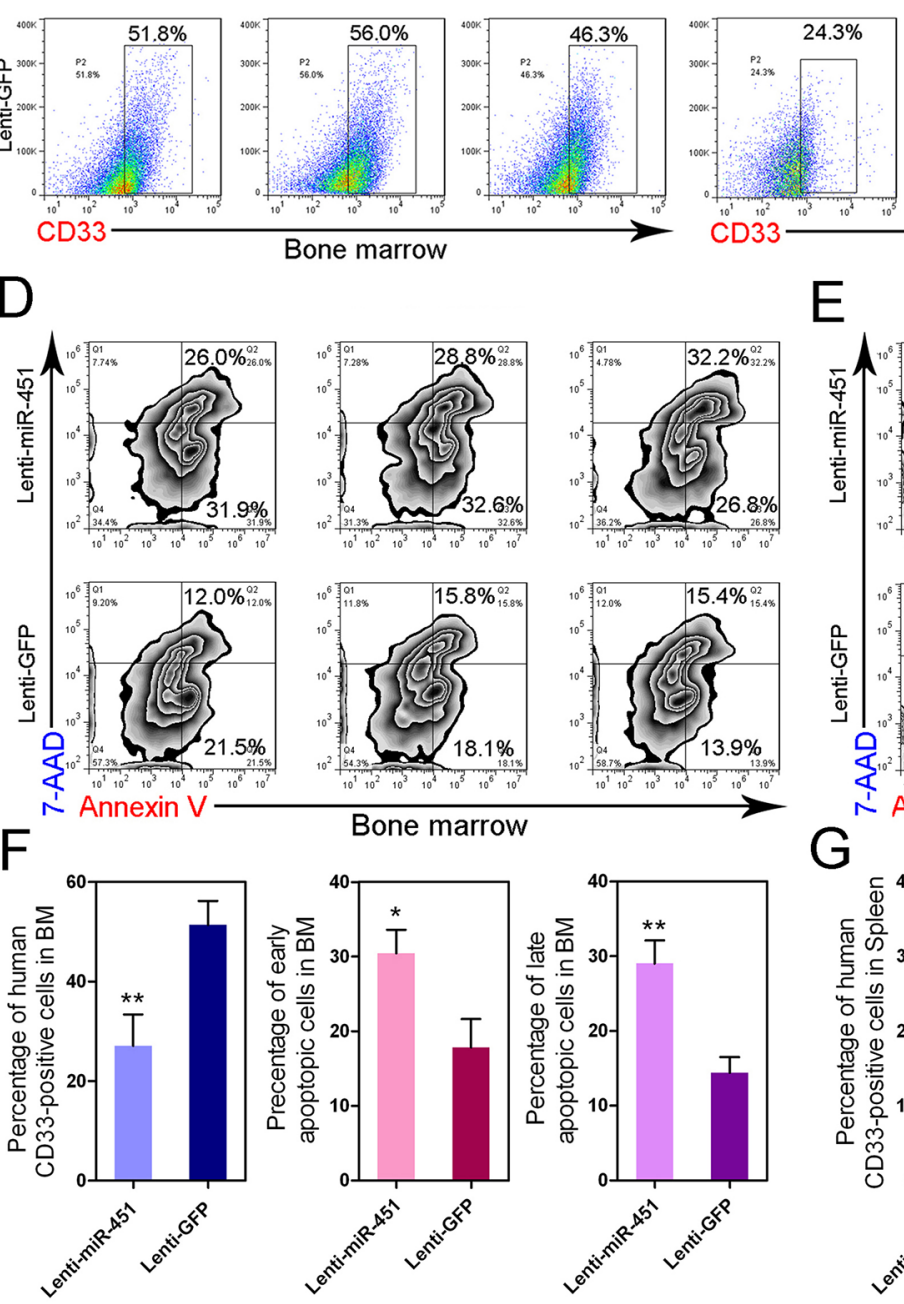

$E$
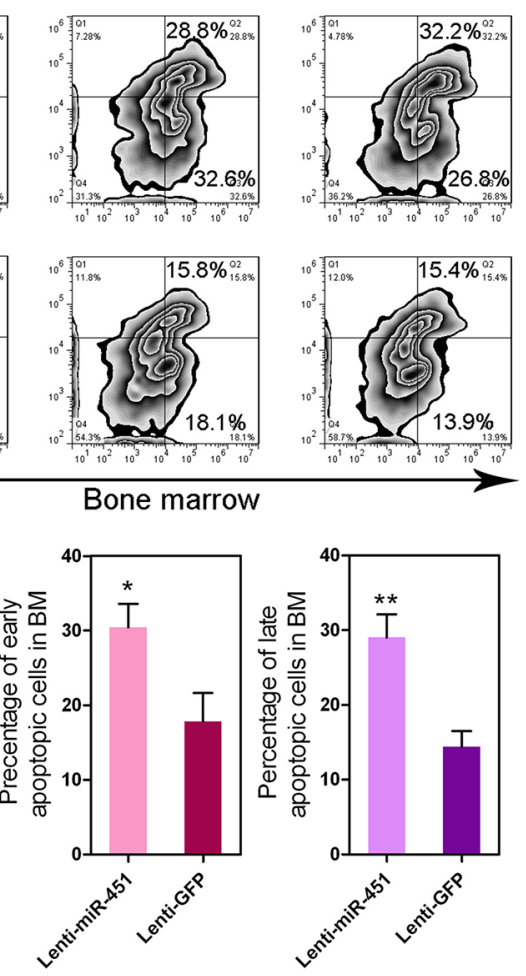

\section{G}
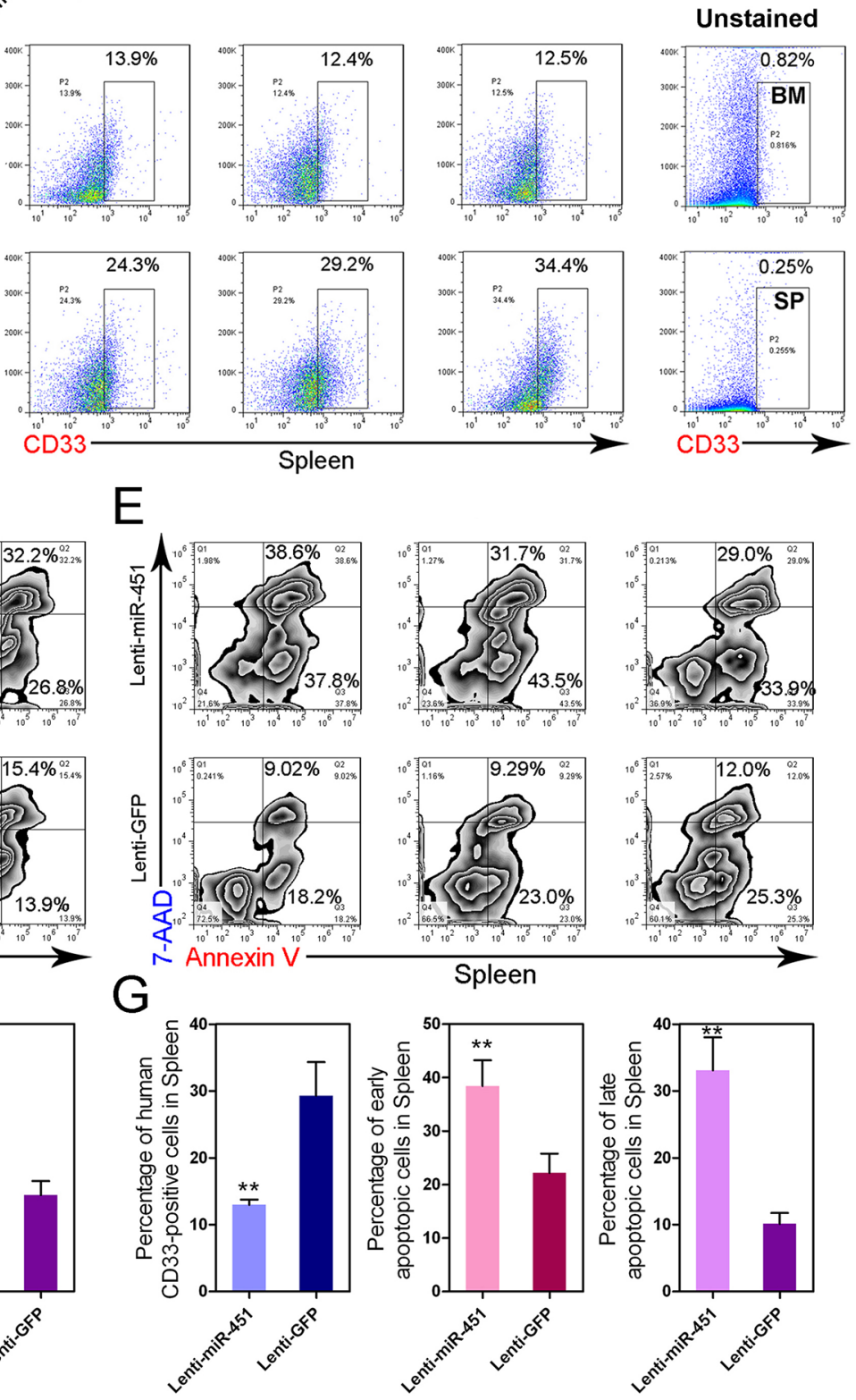

Figure 4: Reintroduction of miR-451 inhibits leukemic spreading and induces apoptosis in AML mouse model. A. Enforced expression of miR-451 relieves splenomegaly induced by leukemia in vivo (left panel). Overexpression of miR-451 was confirmed by qRT-PCR in BM (middle panel) and spleen (right panel). B. H \& E staining of spleen from indicated groups. C. Enforced expression of miR-451 suppressed engraftment of leukemic cells into BM and spleen. The human-specific CD33 surface marker was detected by flow cytometry to identify the human leukemia cells. D and E. Reintroduction of miR-451 induces cell apoptosis in BM (D) and spleen (E). F and G. The statistical results of flow cytometry data, which strongly suggest that reintroduction of miR-451 inhibits engraftment of leukemic cells and induces apoptosis in vivo. ${ }^{*} P<0.05 ; * * P<0.01$, Student's t-test. 


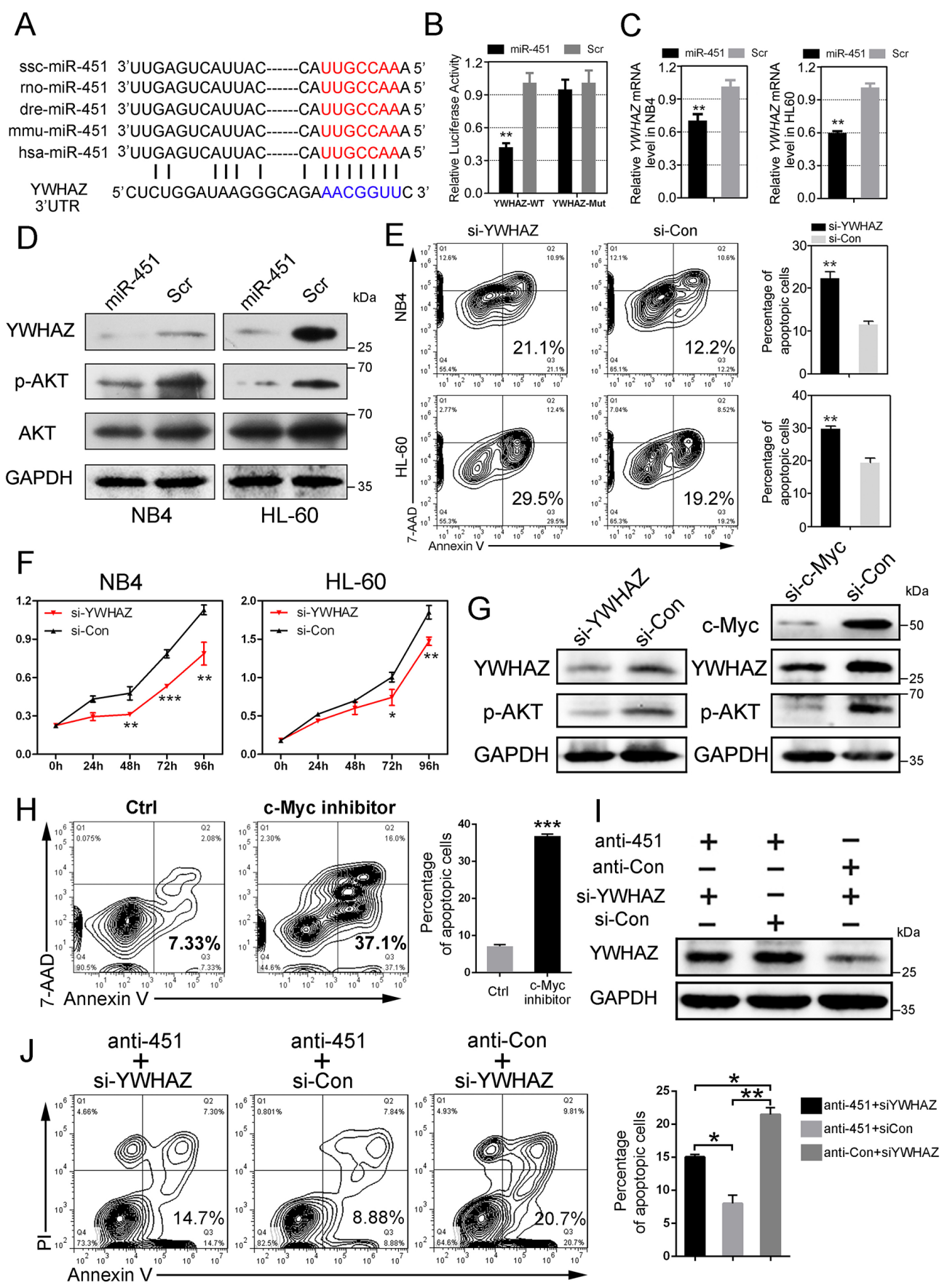

Figure 5: miR-451 directly targets $Y W H A Z$ and subsequently suppresses YWHAZ-AKT signaling in AML cells. A. The predicated potential binding site of miR-451 on the 3'UTR of YWHAZ. The "seed sequence" of miR-451 is marked with red color. B. Enforced miR-451 expression inhibited relative luciferase activity of the construct containing the wild type YWHAZ 3'UTR, but not the mutant. The dual-luciferase reporter assay was performed in triplicate in HEK-293 cells. Error bars represent $\mathrm{SD}(\mathrm{n}=3)$; ** $P<0.01$; Student's t-test. C. Overexpression of miR-451 reduced $Y W H A Z$ mRNA level in NB4 and HL-60 cells. ${ }^{* *} P<0.01$. D. Enforced miR451 expression reduced YWHAZ and subsequently decreased p-AKT protein levels in NB4 and HL-60 cells. E. knockdown of YWHAZ promoted apoptosis of NB4 and HL-60 cells. A representative experiment is presented on the left and a statistical analysis of three independent experiments on the right. ${ }^{*} P<0.01$. F. Knockdown of $Y W H A Z$ restrains proliferation of NB4 and HL-60 cells. G. Either knockdown of YWHAZ or knockdown of $c$-Myc inhibited activity of YWHAZ/AKT signaling in the AML cells. H. c-Myc inhibitor (JQ-1) treatment accelerated apoptosis in NB4 AML cells. A representative experiment is presented on the left and a statistical analysis of three independent experiments on the right. $* * * P<0.001$. I. Immunoblotting of YWHAZ in the NB4 cells that were transfected with anti-451 (or anti-Con) for $24 \mathrm{~h}$ and then treated for another $48 \mathrm{~h}$ with si-YWHAZ (or si-Con). J. The cell apoptosis inhibition by anti-451 transfection can be rescued by re-transfection with si-YWHAZ. A representative experiment is presented on the left and a statistical analysis of three independent experiments on the right. $* P<0.05 ; * P<0.01$. 
5I). Consistent with the YWHAZ expression, the cell apoptosis inhibition by anti-451 could be rescued by retransfection with si-YWHAZ (Figure 5J). Overall, our data suggested that miR-451 directly targets YWHAZ-AKT signaling to regulating cell apoptosis and proliferation in the AML cells.

\section{Abnormal overexpression of both c-Myc and YWHAZ is negatively correlated with miR-451 level in AML patients}

We randomly selected 29 AML patients and 24 healthy donors, whose peripheral blood samples are available for being used for more analyses, to detect expression of c-Myc and YWHAZ by Western blot (Figure 6A). The results displayed that c-Myc and YWHAZ were abnormally up-regulated in most of the detected AML patients as compared to the normal controls (Figure 6B and 6C), while miR-451 was abnormally down-regulated (Figure 6D). We then analyzed the correlation between c-Myc and miR-451, miR-451 and YWHAZ, as well as c-Myc and YWHAZ. Interestingly, miR-451 level not only negatively correlated with c-Myc expression (Figure $6 \mathrm{E}$ ), but also YWHAZ (Figure 6F), while expression of YWHAZ was positively correlated with c-Myc expression (Figure 6G) in the examined subjects. We did not observe significant differences of c-Myc and YWHAZ levels among different AML FBA subtypes (data not shown). The results further confirmed the c-Myc-ImiR-451-YYWHAZ axis in AML.

\section{DISCUSSION}

Abnormal deregulation of miRNAs and the oncogenic or tumor suppressor function of specific miRNAs have been well identified and investigated in tumors [30, 31] as well as in leukemias [32-34], especially in AML [35]. Becker H. et al. and Whitman $\mathrm{SP}$. et al. reported that miR-451 is underexpressed in AML with NPM1 mutation and FLT3-ITD mutation, and deregulation of miR-451 correlated with adverse prognosis in AML with FLT3-ITD mutation $[36,37]$. In this study, we find that miR-451 is aberrantly down-regulated in AML patients, and our in vivo and in vitro data strongly indicates that miR-451 functions as a tumor suppressor through directly increasing cell apoptosis and decreasing cell proliferation in AML.

The proto-oncogene $c-M y c$ encodes a transcription factor whose expression is finely regulated during hematopoiesis and frequently hyper-activated in AML $[38,39]$. High level of c-Myc functions as a transcriptional amplifier to elevate expression of the already transcripted genes [40, 41]. Strikingly, we identified that hyper-activation of c-Myc results in de-regulation of miR-451 in AML. HDACs regulate gene expression by modifying epigenetic configuration via removing acetyl groups from histone tails, and usually highly expressed in leukemia [14, 42]. Presently, we find c-Myc can recruit HDAC3 on the miR-451 promoter and consequently induce its deregulation in AML. Importantly, knockdown of $c-M y c$ or $H D A C 3$ or treatment with their inhibitors (TSA, HDACs inhibitor; JQ-1, c-Myc inhibitor) results in restoration of $\mathrm{miR}-451$ and mimic its functions in AML. All these findings address that miR-451 repression is a result of c-Myc/HDAC3 interaction in AML.

As critical functional targets of miR-451, we identify that miR-451 directly suppresses YWHAZ (14-3$3 \zeta)$ in AML. Inhibition of $Y W H A Z$ could mimic all the functions of miR-451 overexpression. YWHAZ plays a critical role in signaling transduction through interaction with target proteins via phosphorylation motif [43] or directly regulating phosphorylation $[44,45]$. We identify that forced expression of miR-451 or knockdown of $Y W H A Z$ or $c-M y c$ result in decreased p-AKT levels in AML. To address whether the c-Myc-lmiR-451-YYWHAZ regulatory loop is relevant to leukemogenesis, we analyzed the correlation among c-Myc, miR-451 and YWHAZ in AML patients. Consistent our hypothesis, low expression of miR-451 is negatively related with high expression of c-Myc as well as YWHAZ, while c-Myc is positively correlated with YWHAZ level in AML patients.

Collectively, our study led to the identification of a model for interplay among c-Myc, miR-451, YWHAZ and AKT, as well as their contribution to leukemogenesis (Figure 7). Reintroduction of miR-451 or combination of c-Myc and HDAC inhibitors could be a potential strategy for AML therapy.

\section{MATERIALS AND METHODS}

\section{Cell lines and their maintenance}

The human promyelocytic cell line NB4 and human embryonic kidney cell line HEK-293T were cultured in RPMI-1640 medium (Gibco, BRL, UK) supplemented with 10\% FCS (Gibco), $50 \mathrm{U} / \mathrm{ml}$ penicillin, and $50 \mathrm{mg} /$ $\mathrm{ml}$ streptomycin (Sigma, St. Louis, MO, USA) at $37^{\circ} \mathrm{C}$ in $5 \% \mathrm{CO}_{2}$. The human promyelocytic cell line HL-60 was cultured in IMDM medium (Gibco) supplemented with $10 \% \mathrm{FCS}, 50 \mathrm{U} / \mathrm{ml}$ penicillin, and $50 \mathrm{mg} / \mathrm{ml}$ streptomycin (Sigma) at $37^{\circ} \mathrm{C}$ in $5 \% \mathrm{CO}_{2}$. The lentivirus packaging cell line HEK-293TN was maintained in DMEM medium supplemented with $10 \% \mathrm{FCS}, 50 \mathrm{U} / \mathrm{ml}$ penicillin, and 50 $\mathrm{mg} / \mathrm{ml}$ streptomycin (Sigma). HEK-293T cell line was only used for dual-luciferase reporter assay and packaging lentivirus; while NB4 and HL-60 AML cells were used to identify the function of miR-451, YWHAZ, c-Myc and HDAC3.

\section{Human samples}

The PB samples and BM samples from normal volunteers and AML patients were obtained from the 303 Hospital in Nanning, and the 307 Hospital in 


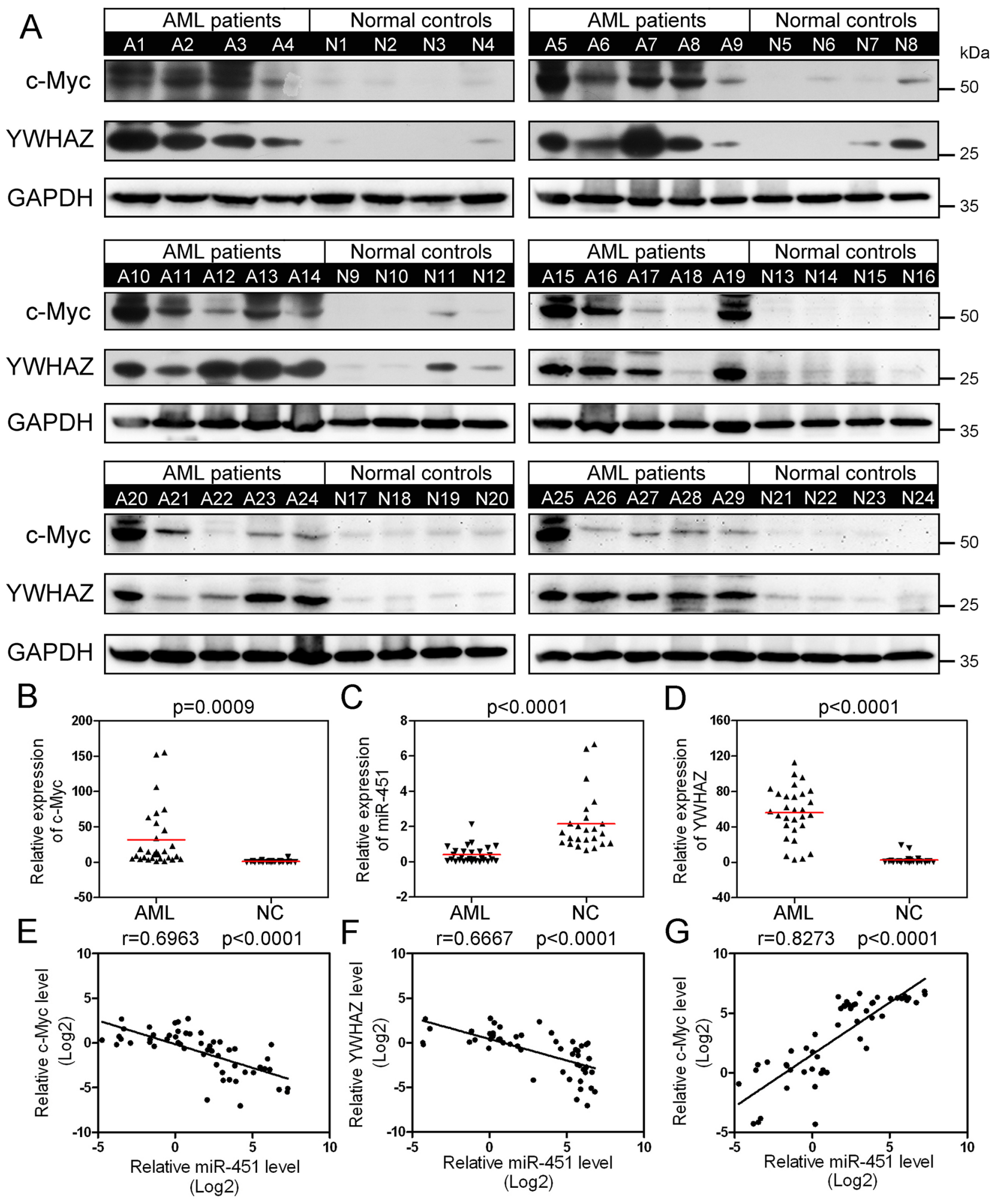

Figure 6: Abnormal upregulation of c-Myc increases expression of YWHAZ via suppressing miR-451 in AML patients. A. Western blot data indicate that c-Myc and YWHAZ are aberrantly up-regulated in almost all the examined AML patient PB MNC samples. B-D. The relative level of c-Myc (B), YWHAZ (C) and miR-451 (D) in the 29 AML patients and 24 healthy donors. The protein levels were quantified using the Image J software and normalized to glyceraldehyde 3-phosphate dehydrogenase (GAPDH). E-G. Pearson correlation analysis between c-Myc and miR-451 levels (E), miR-451 and YWHAZ levels (F), as well as c-Myc and YWHAZ levels (G) in the PBMNC samples. The Pearson correlation coefficient $r$ was calculated and verified by the two-tailed significance test. 
Beijing, China. The informed consent to perform the biological studies was obtained from all of the examined subjects and the related study was approved by the Ethic Committees of the Institutional Review Board of Institute of Basic Medical Sciences (IBMS), Chinese Academy of Medical Sciences (CAMS). The MNCs were isolated from $\mathrm{PB}$ and $\mathrm{BM}$ cells by Percoll density gradient $[\mathrm{d}=1.077]$ (Amersham Biotech, Germany). The CD34 ${ }^{+}$ HSPCs were enriched from BM MNCs through positive immunomagnetic selection (CD34 MicroBead Kit, human, Miltenyi Biotech, Bergisch-Glad-bach, Germany) following the manufacturer's instructions.

\section{Co-Immunoprecipitation (Co-IP) assay}

Co-IP assay were performed as described previously [46]. Antibodies for c-Myc, IgG, HDAC3 and HDAC1 were purchased from Cell Signaling Technology (Danvers, MA, USA).

\section{Chromatin Immunoprecipitation (ChIP)}

$2 \times 10^{7}$ NB4 or HL-60 cells for each immunoprecipitation reaction were collected and cross-linked with $1 \%$ formaldehyde for $10 \mathrm{~min}$ at room temperature. Then chromatin was sonicated to obtain chromatin fragments between 200 and $1000 \mathrm{bp}$. Immunoprecipitation was performed after overnight incubation with anti-c-Myc or anti-HDAC3 (Cell Signaling Technology) or IgG antibody (Cell Signaling Technology) and subsequent incubation with Protein A agarose (Roche). After reverse cross-linking and DNA purification, the input and the immunoprecipitated DNA samples were used as templates to amplify the target

\section{Normal}

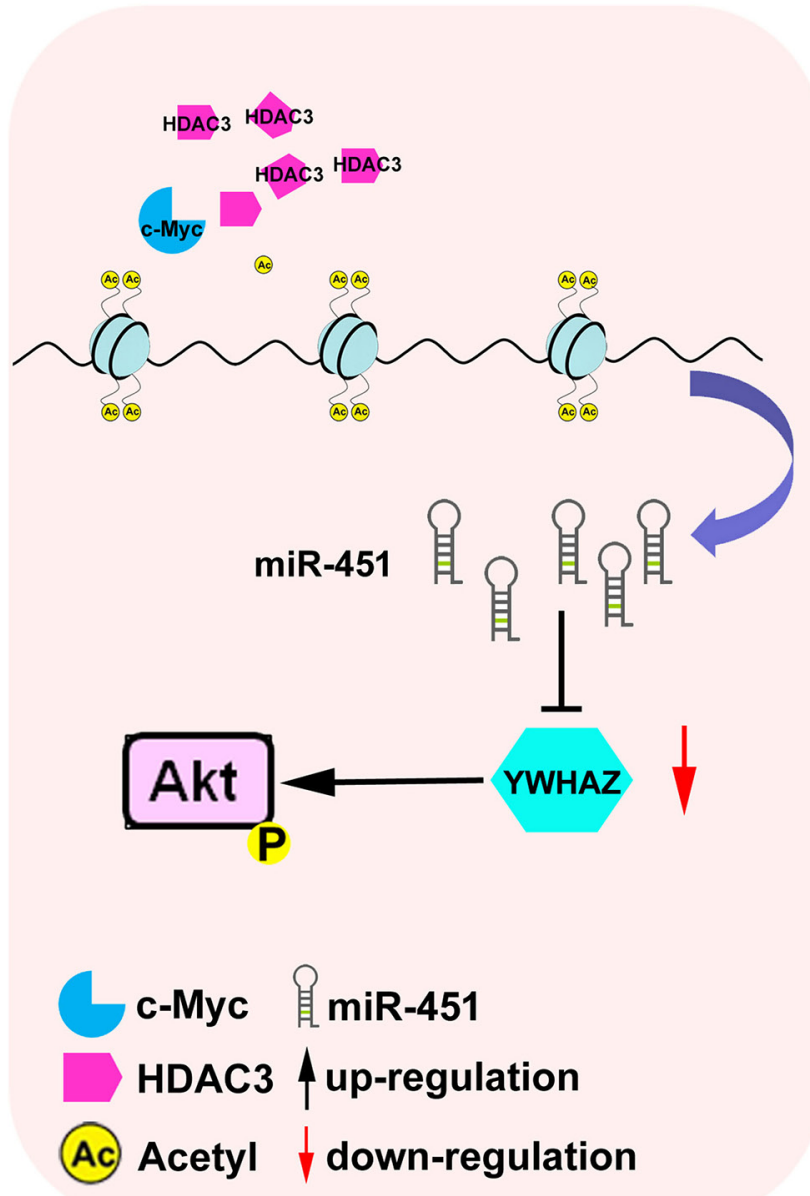

AML

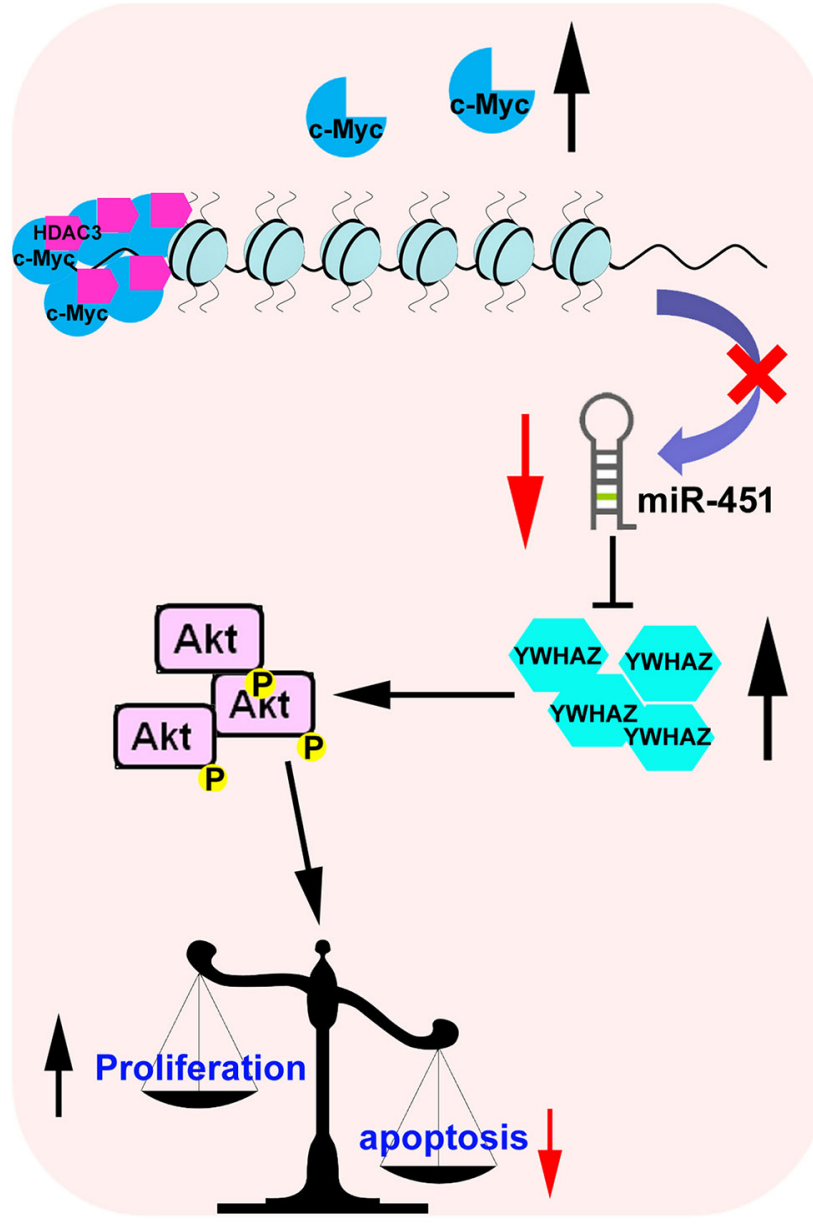

Figure 7: A model for c-Myc/miR-451/YWHAZ/cAKT cascade's being involved in AML development. In normal cells, the relative lower level of c-Myc could maintain expression of miR-451 to inhibit oncogenic YWHAZ/AKT signal. While in AML cells, aberrant overexpression of c-Myc could recruit more HDAC3 on the miR-451 promoter and significantly restrict its expression, which subsequently activates the oncogenic YWHAZ/AKT signal and disturb the balance between proliferation and apoptosis. 
sequences by PCR. The primer sequences are: forward, 5' CCCTGGGTCCCTATGAGATC 3'; reverse, 5' CATGGCTTGAAAAGCACTGTG 3'.

\section{Dual luciferase reporter assay}

For $m i R-451$ promoter activity analysis, a $2703 \mathrm{bp}$ DNA fragment containing the $m i R-451$ promoter region was amplified using human genomic DNA as template and the primers: forward, 5' GTCACTTGGGACCTGTCACCTC 3' ; reverse, 5' CTCAGTAATGGTAACGGTTTCCTTG 3'. The fragment containing the wild type and the fragment with deletion of c-Myc binding site, were respectively inserted into pGL3 basic vector (Promega, WI, USA). The c-Myc ORF was amplified using human genomic DNA as template and the primers: forward, 5' GCCACCATGGATTTTTTTCGGGTAGTG-3'; reverse, 5'-TACATTATGGCTAAATCTTTCAGTCTC-3'. The amplified fragment was inserted into pcDNA3.1. These constructs as well as pRL-TK were transfected into HEK293T cell together, using Lipofectamine 2000 (Invitrogen, CA, USA). The plasmid pRL-TK containing Renilla luciferase was used as internal control. For miR-451 target analysis, the 3'-UTR of human YWHAZ containing the wild type and mutant miR-451 binding site was inserted into pMIR-REPORT. Mutations of the predicted seed regions in YWHAZ mRNA sequence were created using the primers including the mutated sequences. HEK-293T cells were co-transfected with $0.4 \mu \mathrm{g}$ pMIR-REPOTR-YWHAZ construct, $0.02 \mu \mathrm{g}$ pRL-TK control, and 5 pmol of miR451 mimic or scrambled controls. Cells were harvested $48 \mathrm{~h}$ post-transfection and luciferase activity was assayed with Dual-luciferase reporter assay system according to the manufacturer's protocol (Promega). All transfection assays were carried out in triplicate.

\section{RNA isolation, reverse transcription (RT) and quantitative PCR (qPCR)}

Total RNA was isolated from the harvested cells using TRIzol reagent (Invitrogen, Carlsbad, CA, USA) and quantified by absorbance at $260 \mathrm{~nm}$. cDNA was synthesised by M-MLV reverse transcriptase (Invitrogen) from $0.1-1 \mu \mathrm{g}$ of total RNA. Stem-poop RT primers were used for the reverse transcription of miR-451 using the primer 5'- GTCGTATCCAGTGCAGGGTCCGA GGTATTCGCACTGGATACGACAACTCAG-3'. U6 snRNA RT primer is 5'-AAAATATGGAACGCTTC ACGAATTTG-3'; Oligo18 [10] was used for reverse transcription of mRNAs. q-RT-PCR was carried out in Bio-Rad IQ5 real-time PCR System (Bio-Rad, Foster City, CA, USA) using the SYBR Premix Ex Taq kit (Takara, Dalian, China) according to the manufacturer's instruction. Each assay was performed in triplicate. The data were normalized using the endogenous GAPDH for mRNA and U6 snRNA for miRNAs. The primer sequences used for qRT-PCR were: miR-451 forward, 5'CTGGAGAAACCGTTACCATTAC-3'; miR-451 reverse, 5'- GTGCAGGGTCCGAGGT-3'; U6 snRNA forward, 5'-CTCGCTTCGGCAGCACATATACT-3'; U6 snRNA reverse, 5'-ACGCTTCACGAATTTGCGTGTC-3'; YWHAZ forward, 5'-TGATCCCCAATGCTTCACAA G-3'; YWHAZ reverse, 5'-GCCAAGTAACGGTAGTA ATCTCC-3'; GAPDH forward, 5'-TCAACGACCACTTT GTCAAGCTCA-3', GAPDH reverse, 5'-GCTGGTGG TCCAGGGGTCTTACT-3'.

\section{Cell proliferation and apoptosis}

Cell proliferation was determined using the Cell Counting Kit-8 (CCK-8, Dojindo, Kumamoto, Japan) according to the manufacturer's instructions. NB4 and HL-40 cells were seeded into 96 -well plates by 10000 cells/well. At the indicated time points, $10 \mu \mathrm{L}$ CCK-8 was added and the cells were incubated for $3 \mathrm{~h}$ at $37^{\circ} \mathrm{C}$. The optical density was read at $450 \mathrm{~nm}$ with a microplate spectrophotometer. Each experiment was performed in triplicate. For apoptosis, the cells were collected, washed once with PBS and resuspended in the $1 \mathrm{X}$ binding buffer. Apoptotic cells were assessed using the PE Annexin V Apoptosis Detection Kit 1 (BD Bioscience, San Diego, CA, USA), and immediately analyzed by flow cytometry.

\section{Western blot}

Western blot was performed as described previously [47]. The following antibodies were used. Anti-GAPDH and Anti-YWHAZ were purchased from Proteintech Company (Chicago, IL, USA). Anti-HDAC3, AntiHDAC1, Anti-p-AKT, Anti-AKT, Anti-c-Myc and AntiIgG were purchased from Cell Signaling Technology.

\section{Oligonucleotides, cell transfection and drug treatment}

The miR-451 mimics, miR-451 inhibitor, si YWHAZ, si_c-MYC, si_HDAC3 and their negative controls were purchased from Dharmacon (Austin, TX, USA) and transfected into the AML cells with DharmFECT1 (Dharmacon) at a final concentration of $100 \mathrm{nM}$. Six hours later, the medium was changed and cells were harvested at indicated time points for analyses. TSA (HDACs inhibitor) and JQ-1 (c-Myc inhibitor) were purchased from Sigma. For apoptosis assay, NB4 AML cells were treated with $300 \mathrm{nM}$ JQ-1.

\section{Recombination lentivirus production}

A 500 bp DNA fragment containing pre-miR-451 was obtained by PCR amplification from human genome DNA with primers: Forward, 5'-CGGAATTCCCCT GGCTGGGATATCATCATATA-3', Reverse, 5'- TTG CGGCCGCGTATCTATTCCCTCCCCTACCCC-3'. 
The amplified fragment was inserted into downstream of CMV promoter in pMIRNA1 vector. The lentivirus production and infection are the same as described previously [47].

\section{Mouse xenograft assay}

All experiments involving animals were performed according to protocols approved by the Institutional Animal Care and Use committee of IBMS, CAMS. The mouse xenograft assay was performed as described [47] with some modification. Briefly, $1 \times 10^{6} \mathrm{NB} 4$ cells were injected into 4-6 weeks old sublethally irradiated (250cGy) NOD/SCID mice by tail vein and then $1 \times 10^{7}$ lentiviral particles were delivered into the mice once they show typical leukemic symptoms. For mice in the "untreated" group, $100 \mu \mathrm{L}$ EDTA-PBS were injected. About three weeks later, all the mice were euthanized and the tissues including spleen and BM obtained by femur flushing were collected for further detection. Single cell suspension from spleen and BM was prepared for apoptotic analysis as described above and flow cytometric analysis. Murine spleen tissue was fixed in $10 \%$ formalin for $24 \mathrm{~h}$, embedded in paraffin, cut into $4-\mu \mathrm{m}$-thick sections and stained with haematoxylin and eosin (H \& E).

\section{Flow cytomety analysis}

For detecting engraftment of human NB4 cells into murine BM and spleen, the single cell suspension was washed with chilled PBS and suspended in PBS with $0.5 \%$ BSA to block Fc receptors, and were then incubated with PE-conjugated anti-CD33 (BioLegend, San Diego, CA, USA) on ice for $30 \mathrm{~min}$. Finally, cells were washed using cold PBS and fixed in 4\% paraform for further analysis on Accuri C6 flow cytometer (BD, SD, USA).

\section{Statistics}

Student's t-test (two-tailed) was performed to analyze the data. The correlations among relative level of c-Myc, miR-451 and YWHAZ were examined by Pearson correlation analysis by GraphPad Prism 5.0. P-values < 0.05 were considered significantly.

\section{ACKNOWLEGMENTS}

This work was supported by the National Natural Science Foundation of China 31171311 and 30970616 to J-WZ.

\section{CONFLICTS OF INTEREST}

The authors declare no competing financial interests.

\section{REFERENCES}

1. Lowenberg B, Downing JR and Burnett A. Acute myeloid leukemia. N Engl J Med. 1999; 341:1051-1062.

2. Garzon R, Heaphy CE, Havelange V, Fabbri M, Volinia S, Tsao T, Zanesi N, Kornblau SM, Marcucci G, Calin GA, Andreeff $M$ and Croce CM. MicroRNA 29b functions in acute myeloid leukemia. Blood. 2009; 114:5331-5341.

3. Khaled S, Al Malki M and Marcucci G. Acute Myeloid Leukemia: Biologic, Prognostic, and Therapeutic Insights. Oncology. 2016; 30:318-329.

4. Lobry C, Oh P, Mansour MR, Look AT and Aifantis I. Notch signaling: switching an oncogene to a tumor suppressor. Blood. 2014; 123:2451-2459.

5. Tasian SK, Teachey DT and Rheingold SR. Targeting the PI3K/mTOR Pathway in Pediatric Hematologic Malignancies. Front Oncol. 2014; 4:108.

6. Pulikkan JA, Dengler V, Peer Zada AA, Kawasaki A, Geletu M, Pasalic Z, Bohlander SK, Ryo A, Tenen DG and Behre G. Elevated PIN1 expression by C/EBPalpha-p30 blocks C/EBPalpha-induced granulocytic differentiation through c-Jun in AML. Leukemia. 2010; 24:914-923.

7. Kollmann K, Heller G, Ott RG, Scheicher R, ZebedinBrandl E, Schneckenleithner C, Simma O, Warsch W, Eckelhart E, Hoelbl A, Bilban M, Zochbauer-Muller S, Malumbres M, et al. c-JUN promotes BCR-ABL-induced lymphoid leukemia by inhibiting methylation of the $5^{\prime}$ region of Cdk6. Blood. 2011; 117:4065-4075.

8. Salvatori B, Iosue I, Djodji Damas N, Mangiavacchi A, Chiaretti S, Messina M, Padula F, Guarini A, Bozzoni I, Fazi F and Fatica A. Critical Role of c-Myc in Acute Myeloid Leukemia Involving Direct Regulation of miR-26a and Histone Methyltransferase EZH2. Genes \& cancer. 2011; 2:585-592. doi: 10.1177/1947601911416357.

9. Jiang X, Huang H, Li Z, Li Y, Wang X, Gurbuxani S, Chen P, He C, You D, Zhang S, Wang J, Arnovitz S, Elkahloun A, et al. Blockade of miR-150 maturation by MLL-fusion/ MYC/LIN-28 is required for MLL-associated leukemia. Cancer Cell. 2012; 22:524-535.

10. Muller-Tidow C, Steffen B, Cauvet T, Tickenbrock L, Ji P, Diederichs S, Sargin B, Kohler G, Stelljes M, Puccetti E, Ruthardt M, deVos S, Hiebert SW, et al. Translocation products in acute myeloid leukemia activate the Wnt signaling pathway in hematopoietic cells. Mol Cell Biol. 2004; 24:2890-2904.

11. Luo H, Li Q, O'Neal J, Kreisel F, Le Beau MM and Tomasson MH. c-Myc rapidly induces acute myeloid leukemia in mice without evidence of lymphoma-associated antiapoptotic mutations. Blood. 2005; 106:2452-2461.

12. Sarkar D, Leung EY, Baguley BC, Finlay GJ and AskarianAmiri ME. Epigenetic regulation in human melanoma: past and future. Epigenetics. 2015; 10:103-121.

13. Zhang H, Shang YP, Chen HY and Li J. Histone deacetylases function as a novel potential therapeutic target for cancer. Hepatol Res. 2016; doi: 10.1111/hepr. 12757. 
14. Van Damme M, Crompot E, Meuleman N, Mineur P, Bron $\mathrm{D}$, Lagneaux L and Stamatopoulos B. HDAC isoenzyme expression is deregulated in chronic lymphocytic leukemia B-cells and has a complex prognostic significance. Epigenetics. 2012; 7:1403-1412.

15. Nebbioso A, Clarke N, Voltz E, Germain E, Ambrosino C, Bontempo P, Alvarez R, Schiavone EM, Ferrara F, Bresciani F, Weisz A, de Lera AR, Gronemeyer H, et al. Tumor-selective action of HDAC inhibitors involves TRAIL induction in acute myeloid leukemia cells. Nat Med. 2005; 11:77-84.

16. Stumpel DJ, Schneider P, Seslija L, Osaki H, Williams O, Pieters R and Stam RW. Connectivity mapping identifies HDAC inhibitors for the treatment of $t(4 ; 11)$-positive infant acute lymphoblastic leukemia. Leukemia. 2012; 26:682-692.

17. Suresh PS, Devaraj VC, Srinivas NR and Mullangi R. Review of bioanalytical assays for the quantitation of various HDAC inhibitors such as vorinostat, belonistat, panobinostat, romidepsin and chidamine. Biomed Chromatogr. 2016; doi: 10.1002/bmc.3807.

18. Musilova K and Mraz M. MicroRNAs in B-cell lymphomas: how a complex biology gets more complex. Leukemia. 2015; 29:1004-1017.

19. Ahmad N, Haider S, Jagannathan S, Anaissie E and Driscoll JJ. MicroRNA theragnostics for the clinical management of multiple myeloma. Leukemia. 2014; 28:732-738.

20. Undi RB, Kandi R and Gutti RK. MicroRNAs as Haematopoiesis Regulators. Adv Hematol. 2013; 2013:695754.

21. Rasmussen KD, Simmini S, Abreu-Goodger C, Bartonicek N, Di Giacomo M, Bilbao-Cortes D, Horos R, Von Lindern M, Enright AJ and O'Carroll D. The miR-144/451 locus is required for erythroid homeostasis. J Exp Med. 2010; 207:1351-1358.

22. Pase L, Layton JE, Kloosterman WP, Carradice D, Waterhouse PM and Lieschke GJ. miR-451 regulates zebrafish erythroid maturation in vivo via its target gata2. Blood. 2009; 113:1794-1804.

23. Wang R, Chen DQ, Huang JY, Zhang K, Feng B, Pan BZ, Chen J, De W and Chen LB. Acquisition of radioresistance in docetaxel-resistant human lung adenocarcinoma cells is linked with dysregulation of miR-451/c-Myc-survivin/ rad-51 signaling. Oncotarget. 2014; 5:6113-6129. doi: 10.18632/oncotarget.2176.

24. Li HP, Zeng XC, Zhang B, Long JT, Zhou B, Tan GS, Zeng WX, Chen W and Yang JY. miR-451 inhibits cell proliferation in human hepatocellular carcinoma through direct suppression of IKK-beta. Carcinogenesis. 2013; 34:2443-2451.

25. Bergamaschi A and Katzenellenbogen BS. Tamoxifen downregulation of miR-451 increases 14-3-3zeta and promotes breast cancer cell survival and endocrine resistance. Oncogene. 2012; 31:39-47.
26. Wang J, Elahi A, Ajidahun A, Clark W, Hernandez J, Achille A, Hao JH, Seto E and Shibata D. The interplay between histone deacetylases and c-Myc in the transcriptional suppression of HPP1 in colon cancer. Cancer Biol Ther. 2014; 15:1198-1207.

27. Zhang X, Zhao X, Fiskus W, Lin J, Lwin T, Rao R, Zhang Y, Chan JC, Fu K, Marquez VE, Chen-Kiang S, Moscinski LC, Seto E, et al. Coordinated silencing of MYC-mediated miR-29 by HDAC3 and EZH2 as a therapeutic target of histone modification in aggressive B-Cell lymphomas. Cancer Cell. 2012; 22:506-523.

28. Zhang X, Chen X, Lin J, Lwin T, Wright G, Moscinski LC, Dalton WS, Seto E, Wright K, Sotomayor E and Tao J. Myc represses miR-15a/miR-16-1 expression through recruitment of HDAC3 in mantle cell and other non-Hodgkin B-cell lymphomas. Oncogene. 2012; 31:3002-3008.

29. Lee JJ, Lee JS, Cui MN, Yun HH, Kim HY, Lee SH and Lee JH. BIS targeting induces cellular senescence through the regulation of 14-3-3 zeta/STAT3/SKP2/p27 in glioblastoma cells. Cell Death Dis. 2014; 5:e1537.

30. Calin GA and Croce CM. MicroRNA signatures in human cancers. Nat Rev Cancer. 2006; 6:857-866.

31. Lin S and Gregory RI. MicroRNA biogenesis pathways in cancer. Nat Rev Cancer. 2015; 15:321-333.

32. Pekarsky Y and Croce CM. Role of miR-15/16 in CLL. Cell Death Differ. 2015; 22:6-11.

33. Liu J, Chen G, Feng L, Zhang W, Pelicano H, Wang F, Ogasawara MA, Lu W, Amin HM, Croce CM, Keating MJ and Huang P. Loss of p53 and altered miR15-a/16-1short right arrowMCL-1 pathway in CLL: insights from TCL1Tg:p53(-/-) mouse model and primary human leukemia cells. Leukemia. 2014; 28:118-128.

34. Fabbri M, Garzon R, Andreeff M, Kantarjian HM, GarciaManero G and Calin GA. MicroRNAs and noncoding RNAs in hematological malignancies: molecular, clinical and therapeutic implications. Leukemia. 2008; 22:1095-1105.

35. Li Z, Chen P, Su R, Li Y, Hu C, Wang Y, Arnovitz S, He M, Gurbuxani S, Zuo Z, Elkahloun AG, Li S, Weng H, et al. Overexpression and knockout of miR-126 both promote leukemogenesis. Blood. 2015; 126:2005-2015.

36. Becker H, Marcucci G, Maharry K, Radmacher MD, Mrozek K, Margeson D, Whitman SP, Wu YZ, Schwind S, Paschka P, Powell BL, Carter TH, Kolitz JE, et al. Favorable prognostic impact of NPM1 mutations in older patients with cytogenetically normal de novo acute myeloid leukemia and associated gene- and microRNA-expression signatures: a Cancer and Leukemia Group B study. Journal of clinical oncology. 2010; 28:596-604.

37. Whitman SP, Maharry K, Radmacher MD, Becker H, Mrozek K, Margeson D, Holland KB, Wu YZ, Schwind S, Metzeler KH, Wen J, Baer MR, Powell BL, et al. FLT3 internal tandem duplication associates with adverse outcome and gene- and microRNA-expression signatures in patients 
60 years of age or older with primary cytogenetically normal acute myeloid leukemia: a Cancer and Leukemia Group B study. Blood. 2010; 116:3622-3626.

38. Weng S, Matsuura S, Mowery CT, Stoner SA, Lam K, Ran D, Davis AG, Lo MC and Zhang DE. Restoration of MYCrepressed targets mediates the negative effects of GM-CSF on RUNX1-ETO leukemogenicity. Leukemia. 2016; doi: 10.1038/leu.2016.167.

39. Pippa R, Dominguez A, Malumbres R, Endo A, Arriazu E, Marcotegui N, Guruceaga E and Odero MD. MYCdependent recruitment of RUNX1 and GATA2 on the SET oncogene promoter enhances PP2A inactivation in acute myeloid leukemia. Oncotarget. 2016; doi: 10.18632/ oncotarget.9840.

40. Nie Z, Hu G, Wei G, Cui K, Yamane A, Resch W, Wang R, Green DR, Tessarollo L, Casellas R, Zhao K and Levens D. c-Myc is a universal amplifier of expressed genes in lymphocytes and embryonic stem cells. Cell. 2012; 151:68-79.

41. Lin CY, Loven J, Rahl PB, Paranal RM, Burge CB, Bradner JE, Lee TI and Young RA. Transcriptional amplification in tumor cells with elevated c-Myc. Cell. 2012; 151:56-67.

42. Forsberg EC and Bresnick EH. Histone acetylation beyond promoters: long-range acetylation patterns in the chromatin world. Bioessays. 2001; 23:820-830.
43. Han X, Han Y, Jiao H and Jie Y. 14-3-3zeta regulates immune response through Stat3 signaling in oral squamous cell carcinoma. Mol Cells. 2015; 38:112-121.

44. Brunet A, Bonni A, Zigmond MJ, Lin MZ, Juo P, Hu LS, Anderson MJ, Arden KC, Blenis J and Greenberg ME. Akt promotes cell survival by phosphorylating and inhibiting a Forkhead transcription factor. Cell. 1999; 96:857-868.

45. Neal CL, Xu J, Li P, Mori S, Yang J, Neal NN, Zhou X, Wyszomierski SL and Yu D. Overexpression of 14-3-3zeta in cancer cells activates PI3K via binding the $\mathrm{p} 85$ regulatory subunit. Oncogene. 2012; 31:897-906.

46. Su R, Dong L, Zou D, Zhao H, Ren Y, Li F, Yi P, Li L, Zhu Y, Ma Y, Wang J, Wang F and Yu J. microRNA$23 \mathrm{a},-27 \mathrm{a}$ and -24 synergistically regulate JAK1/Stat3 cascade and serve as novel therapeutic targets in human acute erythroid leukemia. Oncogene. 2016; doi:10.1038/ onc.2016.127.

47. Su R, Lin HS, Zhang XH, Yin XL, Ning HM, Liu B, Zhai PF, Gong JN, Shen C, Song L, Chen J, Wang F, Zhao HL, et al. MiR-181 family: regulators of myeloid differentiation and acute myeloid leukemia as well as potential therapeutic targets. Oncogene. 2015; 34:3226-3239. 